\title{
GIANT OVARIAN CYST IN PREGNANCY
}

\author{
G. $\operatorname{Radu}^{1}$, Nicoleta Prună ${ }^{1}$, S. Prună ${ }^{1}$, Mirela Moarcăș ${ }^{1}$, Adriana Andrei ${ }^{1}$, Dina Mohammad ${ }^{1}$, \\ O. Munteanu ${ }^{1,2}$, Monica Mihaela Cîrstoiu ${ }^{1,2}$ \\ ${ }^{1}$ The Obstetrics and Gynecology Department, Bucharest Emergency University Hospital \\ ${ }^{2}$ The University of Medicine and Pharmacy "Carol Davila” Bucharest, Romania
}

Corresponding author: Gabriel Radu

Phone no. 0040213180519

E-mail: gabriel.radu88@yahoo.com

\begin{abstract}
Before the use of ultrasound, many ovarian tumors remained undiagnosed until cesarean section or until they became symptomatic. Now, many symptomatic or non-symptomatic adnexal tumors are diagnosed by ultrasound during pregnancy follow-up.In most cases, the adnexal tumors discovered during pregnancy are benign with a maximum diameter of $5 \mathrm{~cm}$. Approximately $70 \%$ of the cystic adnexal tumors identified in the first trimester resorb spontaneously until the beginning of the second trimester (usually those are functional cysts). Persistent adnexal tumors larger than $5 \mathrm{~cm}$ are generally mature teratomas (dermoid).In this case, at the emergency room a 34 year-oldwomen came with almost 20 weeks pregnancy, accusing acute abdominal pain, nausea and vomiting. One week before, the patient went for a routine ultrasound for pregnancy and the doctor discovered a giant tumor larger than $15 \mathrm{~cm}$ (due to the size it could not be measured) with fluid content. Two days before the symptoms, the patient went for an abdominal MRI where a voluminous pelvic-abdominal cyst with almost $30 \mathrm{~cm}$ in diameter was discovered. Differential diagnosis: ovarian torsion or ruptured cyst complicating pregnancy. What is the correct diagnosis and what is to be done since there is a 20 weeks pregnancy?
\end{abstract}

Keywords: giant cyst, ovarian tumor, pregnancy, MRI, acute abdominal pain

\section{Introduction}

During a routine ultrasound in pregnancy a transonic cyst was discovered. According to a survey, between 0.2 and $2 \%$ of pregnancies are complicated by tumors with ovarian origin, and under $6 \%$ of these are malignant [1-9].

Before the use of ultrasound, many ovarian tumors remained undiagnosed until cesarean section or until they became symptomatic. Now, many symptomatic or non-symptomatic adnexal tumors are diagnosed by ultrasound during pregnancy follow-up.
In a retrospective study of more than 46,500 term, cesarean procedures performed for obstetrical indications, 151 patients $(0.3 \%)$ underwent surgical excision of an adnexal tumor. Among these, 83 of $151(51.55 \%)$ patients have had the tumor discovered during surgery. In 148 cases the tumor was benign with a histopathological diagnosis of dermoid cyst (24\%), serous cyst (15\%), mucinous cystadenoma $(11 \%)$, serous cystadenoma $(7 \%)$ [10,11].

In most cases, the adnexal tumors discovered during pregnancy are benign with a maximum diameter of $5 \mathrm{~cm}$ [12]. Approximately $70 \%$ of 
the cystic adnexal tumors identified in the first trimester resorb spontaneously until the beginning of the second trimester (usually those are functional cysts) $[14,15]$. Persistent adnexal tumors larger than $5 \mathrm{~cm}$ are generally mature teratomas (dermoid) [10,11].

Correct diagnosis of these tumors can only be established by histopathological analysis but some tumors show pathognomonic features visible by ultrasound (corpus luteum cyst, follicular cyst, endometriosis cyst, dermoid cyst) [13]. It is important that the pathologist who examines the tumor is made aware of the pregnancy due to pregnancy-induced changes that appear on the histological level and that may affect the diagnosis. In one study, 8 out of 10 neoplasms diagnosed during pregnancy were showing microscopic and clinical features suggestive for an aggressive invasive carcinoma, but these features have regressed postpartum, and it was later confirmed that they actually had a low malignant potential [16].

Scheduling surgery during pregnancy is important. It should be done after the first trimester due to the fact that functional cysts would have been resorbed until this moment, organogenesis is complete (risk of teratogen drugs during anesthesia), the function of the corpus luteum was taken over by the placenta and miscarriages due to intrinsec fetal abnormalities are likely to have already occurred and they will not be erroneously attributed to the surgery[17-21].

\section{Presentation of the case}

On $02 / 14 / 2013$ at the emergency room, a 34 year-old-women with almost 20 weeks pregnancy is accusing pelvic and abdominal intense pain, nausea and vomiting started an hour before.

Anamnesis: Smoker ( $1 / 2$ packages of cigarettes / day for 15 years) 0 births, 1 abortion (on request 12 years prior), first day of last period - 9/28/2012 (20 weeks pregnant).

On 12.18.2012 the Double test results: gestational age 12 weeks and 2 days (according to CRL) PAPP- A 2.03 IU / L, Free beta-HCG 27.04 IU / L, PAAP -A MoM 1.23, calculated risk: 1 / 4300. Interpretation: The patient does not belong to a high-risk group for fetal 21 trisomy, negative screening, risk of maternal age related Down syndrome: 1/220.

Blood type: A II, $\mathrm{Rh}$-negative without isoimmunization.

02/04/2013 ultrasonography: Ongoing pregnancy. Intraabdominally, a transonic image can be observed with a fluid content, larger than $15 \mathrm{~cm}$ (the diameter can not be measured by ultrasound). Recommendations: abdominopelvic MRI (Figure 1).

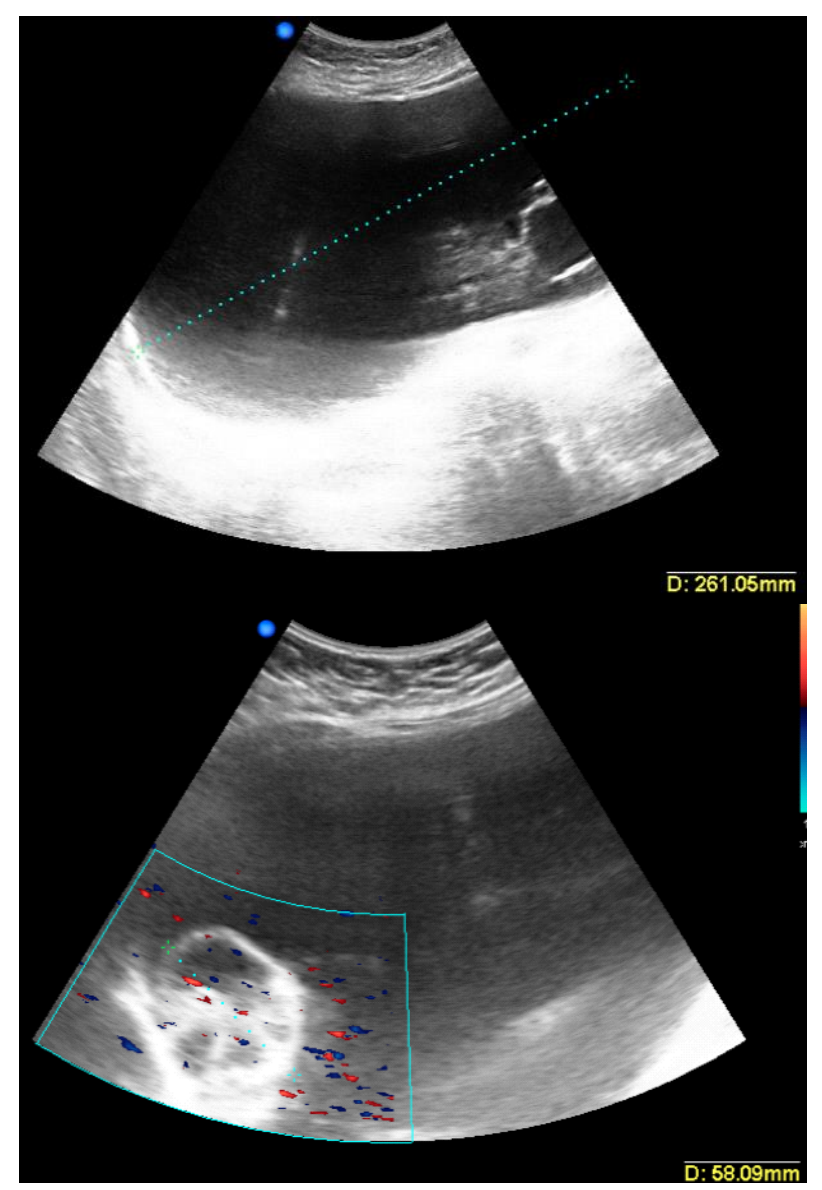

Figure 1 - Ultrasonography - transonic image, with fluid content, larger than $15 \mathrm{~cm}$, fetal parts can also be seen (personal archive)

02/12/2013 pelviabdominal native MRI: voluminous pelvic-abdominal cyst, occupying the mesogastrium and epigastrium with extension in the flanks, next to the uterus, having close contact with the visceral side of the liver, with the gallbladder, the pancreas and the hepatic flexure of the colon, dislodging the intestines and compressing the right ureter, causing right ureterohydronephrosis. The described tumor measures $28 / 23.6 / 15.5 \mathrm{~cm}$, is well encapsulated (in hypo signal T2, T1) and shows in the inside two heterogeneous zones with solid parts (with intermediate signal T2, 
T1) and multilocular cystic parts (hyper signal T2, hypo signal T1) placed inferior and lateral on the left $(12.7 / 11.4 \mathrm{~cm})$ and superior and lateral on the right $(7.5 / 6.3 \mathrm{~cm})$. The described cyst seems to have adnexal origin; its right lower pole is multilocular and has a fat component on the posterior wall (in hyper signal T2, T1, STIR hypo signal, of about $2.5 / 1.6 \mathrm{~cm}$ ). Another multilocular cyst stands out in the left iliac fossa and the left flank, having predominantly lipid densities inside, but also solid densities of $10 / 6.8 \mathrm{~cm}$, with left ovarian affiliation. Enlarged uterus with a single fetus in the cavity $(\mathrm{BPD}=48.9 \mathrm{~mm}=$ pregnancy of approx. 18 weeks) with posterior uterine fibroid of $4.6 / 3 \mathrm{~cm}$ in hypo signal T2. Small fluid film in the pelvic recesses. MRI conclusions: cystic and solid ovarian tumors, with lipid densities inside - dysembryoplasic tumors? Fibroid uterus with single intrauterine fetus of about 18 weeks. Light homogeneous hepatosplenomegaly. Minimal ascites (Figure 2,3,4,5).

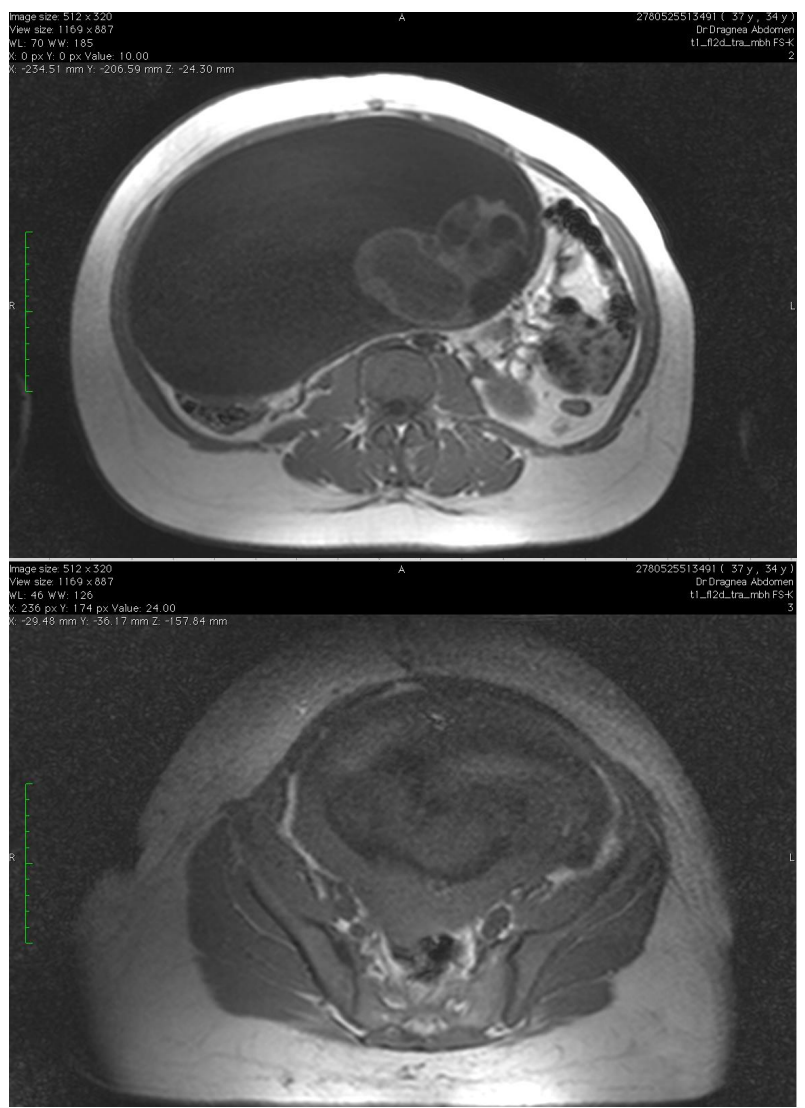

Figure 2 - Pelviabdominal MRI (personal archive) The giant tumor occupying the abdominal cavity and the pregnant uterus

Clinical examination: malaise, faintness, and acute surgical abdomen with muscular defense, spontaneously painful, intensely sensitive to touch. On speculum examination: the patient does not lose blood or AF through the vagina, the cervix is of a nulliparous, without visible lesions. On vaginal examination the Douglas cul-de-sac is found to be intensely painful and the adnexal areas are also painful.

Ultrasonography: a transonic cystic formation is identified, occupying the entire abdomen cavity and an ongoing pregnancy.

Differential Diagnosis: ovarian torsion/ruptured cyst complicating pregnancy

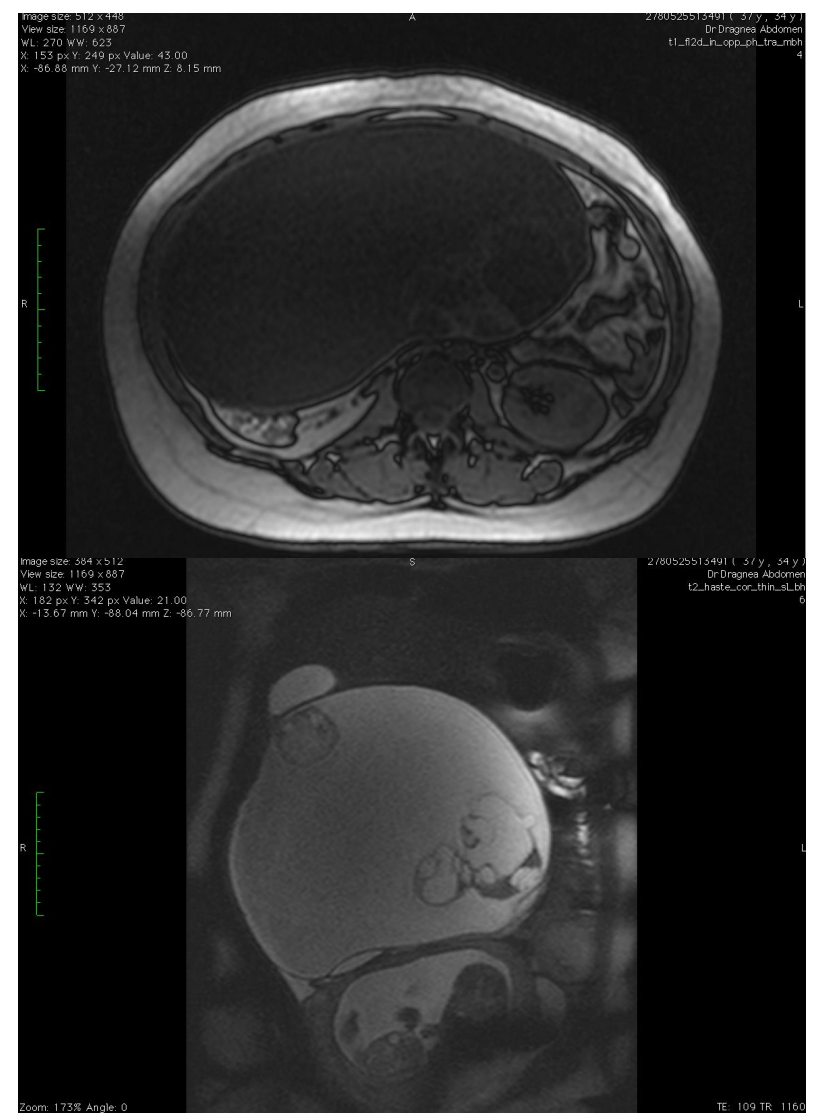

Figure 3 - Pelviabdominal MRI (personal archive) The giant tumor occupying the abdominal cavity and the pregnant uterus

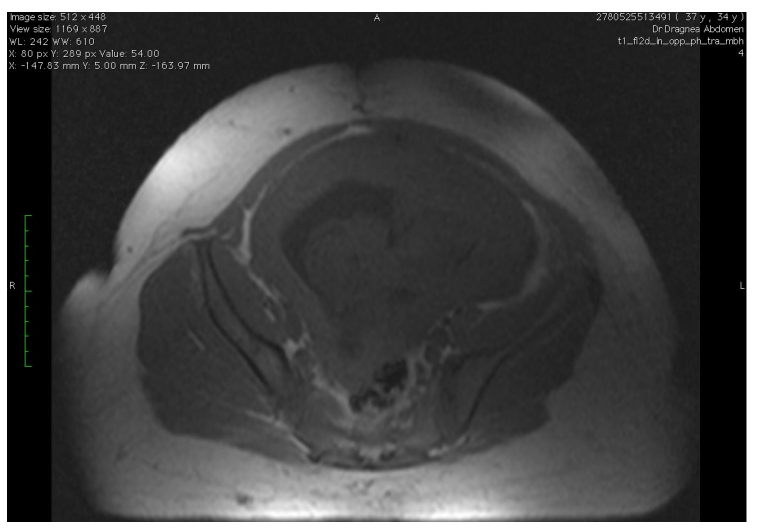

Figure 4 - MRI - fetal parts, head, chest (personal archive) 


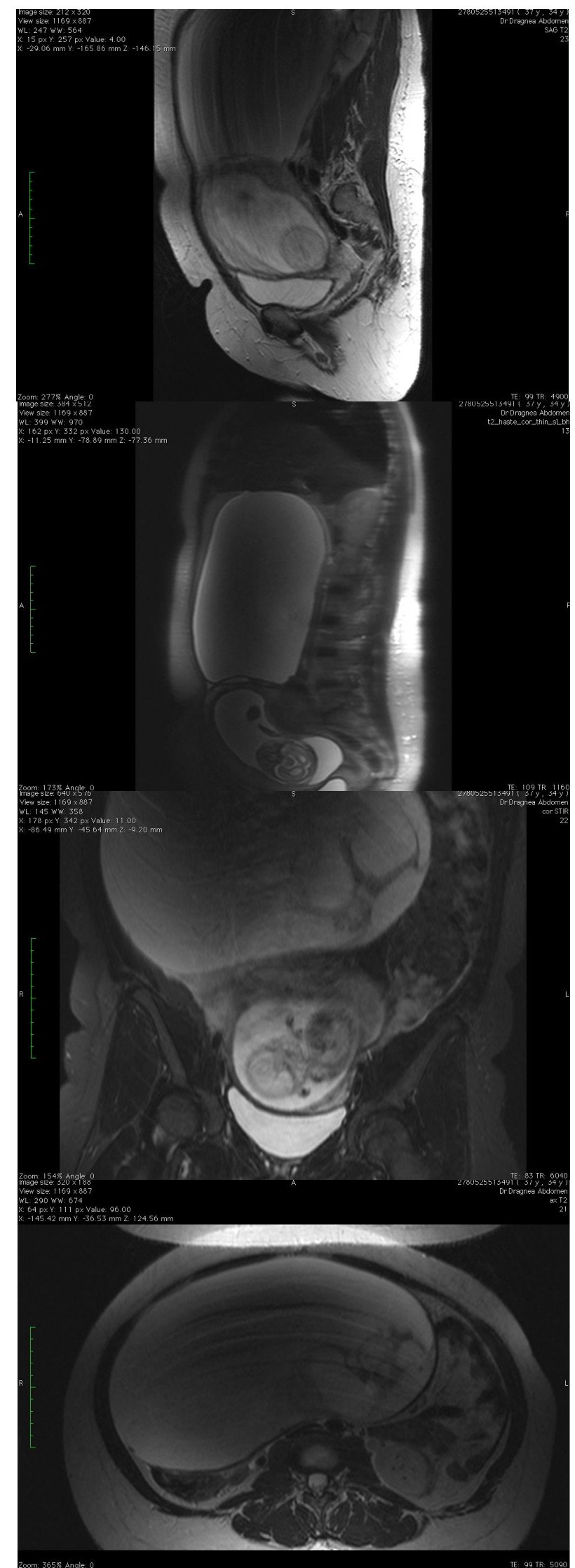

Figure 5 - MRI - pregnant uterus and abdominal tumor

Operative technique: median incision (xifopubian). Intraperitoneally a giant tumor is visualized which occupies the entire peritoneal cavity of about $25-30 \mathrm{~cm}$ long by $20 \mathrm{~cm}$ wide. An incision is made and the fluid vacuumed. The tumor is multilocular. Approximately $4 \mathrm{~L}$ of serosanguinolent liquid are extracted. The increased uterus volume is visualized and the right ovary origin of the tumor. Anexectomy is performed and sent to the histopathological examination. Favorable postoperative evolution, good general condition, afebrile, resumed remission of symptoms.

HP stands out in macroscopic exam: cystic formations with diameter over $25 \mathrm{~cm}$, bleeding inside the cystic wall, containing seborrhea and hair. Histopathological diagnosis: ovarian mucinous cystadenoma twisted, bleeding in the cyst wall. Dermoid cyst.

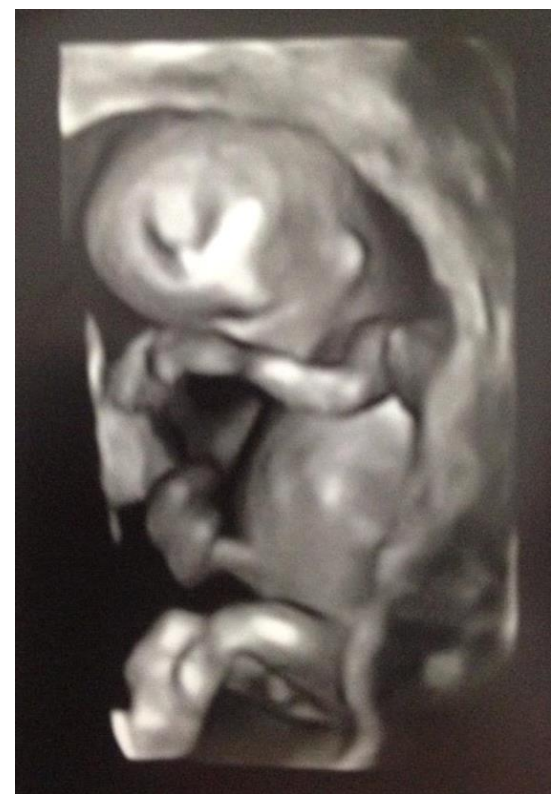

Figure 6 - 3D ultrasound, breech presentation, $21 \mathrm{~W}+3 \mathrm{D}$

Second trimester fetal ultrasound morphology (02/20/2013): Ongoing unique pregnancy with normal fetal movements, breech presentation, AFI 10.7, anterior placenta away from internal orifice. Doppler: increased bilateral uterine artery RI. Gestational age based on last period $20 \mathrm{~W}+6 \mathrm{D}$, Ultrasonography gestational age $21 \mathrm{~W}+3 \mathrm{D}$, boy, $420 \mathrm{~g}$. Conclusion: $21 \mathrm{~W}+3 \mathrm{D}$ ongoing pregnancy, breech presentation. Doppler study: RI increased on uterine artery bilateral (Figure 6).

Ultrasound examinations on $03 / 08 / 2013$ and 05/22/2013 - Ongoing unique pregnancy with normal fetal movements, cranial presentation, boy, normal uterine, umbilical and cerebral artery flow, nuchal cord (Figure 7). 


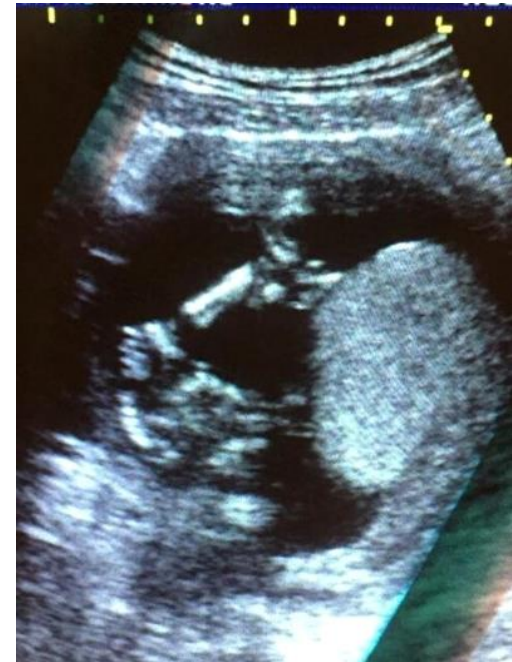

Figure 7 - ultrasound - ongoing pregnancy

On 06/25/2013 at gestational age $38 \mathrm{~W}+5 \mathrm{D}$ cesarean section was performed due to the obstetrical indications. Live boy, G 3150g, Apgar score 9, cephalic presentation with nuchal cord.

\section{Discussions}

The particularity of the case lies in the coexistence of an ovarian tumor and an ongoing pregnancy, a rarely encountered situation according to studies. To the particularity of the case is added the existence of a giant tumor, more than $25 \mathrm{~cm}$ in diameter.

\section{Conclusions}

A giant intraperitoneal tumor may complicate pregnancy causing intrauterine growth restriction, ovarian torsion or a ruptured cyst. In this case the complication was ovarian torsion with acute abdominal pain, nausea and vomiting. The diagnosis was not hard to establish in this case, either ovarian torsion or ruptured cyst. The difficulty of this case consists in the correct decision for treatment. Obviously, surgery was necessary for both of the options: ruptured cyst or ovarian torsion. The decision to leave the ongoing pregnancy had some risks, had the baby growth restriction, will it still grow, will the drugs affect him? After the surgery, the evolution of the patient and the evolution of the pregnancy were both positive and the pregnancy was carried to term with no intrauterine growth restriction.

We want to mention that if the corpus luteum is surgically removed before 8 weeks of pregnancy, administration of progesterone is required after surgery. According to the research done, we did not find any studies demonstrating that pregnancy can affect the prognosis of the ovarian tumors.

\section{References}

[1]Leserowitz GS, Xing G, Cress R, Brahmbhatt B, Dairymple JL, Smith LH - Adnexal masses in pregancy: how often are thei malignant?, Pubmed, Gynecol oncol. 2006;101(2):315.

[2]Schmeler KM, Mayo-Smith WW, Pepert JF, Weitzen S, Maneul MD, Gordinier ME - Adnexal masses in pregnancy: surgery compared with observation, Pubmed, Gynecol Oncol. 2005:105(5pt1):1098.

[3]Smith LH, Dairymple JL, Leiserowitz GS, Danielsen B, Gilbert WM, Obstetrical deliveries associated with maternal malignany in California, 1992 through 1997, Pubmed, Am J Obstet Gynecol.2001;184(7):1504.

[4]Baser E, Erkilinc S, Esin S, Togrul C, Biberoglu E, Karaca MZ, Gungor T, Danisman N, Adnexal masses encountered during cesarean delivery,Pubmed, Int J Gynaecol OBstet. 2013 Nov; 123(2):124:6. Epub 2013 Aug 14.

[5]Runowics CD, Brewer M, Goff B, Falk SJ, Adnexal mass in pregnancy, uptodate.

[6]Giuntoli RL 2nd, Vang RS, Bristow RE. Evaluation and management of adnexal masses during pregnancy. Clin Obstet Gynecol 2006;49:492.

[7]Schmeler KM, Mayo-Smith WW, Peipert JF, et al. Adnexal masses in pregnancy: surgery compared with observation. OBstet Gynecol 2005; 105:1098.

[8]Mooney J, Silva E, Tornos C, Gershenson D. Unusual features of serous neoplasms of low malignant potential during pregnancy. Gynecol Oncol 1997; 64:30.

[9]Gueriero S, Alcazar JL, Ajossa S, et al. Comparison of conventional color Doppler imagind and power doppler imaging for the diagnoisis of ovarian cancer: results of a European study. Gynecol Oncol 2001; 83:299.

[10]Gilman J, Stein H. The corpus luteum of pregnancy. Surg Gynecol Obstet 1941; 72:129.

[11]Clement PB. Tumor-like lesions of the ovary associated with pregnancy. Int J Gynecol Pathol 1993;12:108 
[12]Killackey MA, Neurwirth RS. Evaluation and management of the pelvic mass: a review of 540 cases. Obstet Gynecol 1988;71:319.

[13]Greenlee RT, Kessel B, Williams CR, et al. Prevalnce, incidence, and natural history of simple ovarian cysts among women $>55$ years old in a large cancer screening trial. Am J Obstet Gynecol 2010;202::373.e1.

[14]Modesitt SC, Pavlik EJ, Ueland FR, et al. Risk of malignancy in unilocular ovarian cystic tumors less than 1- cm in diameter. Obstet Gynecol 2003; 102:594.

[15]Wang PH, Chang HT, Yuan CC, et al. Ovarian tumors complicating pregnancy. Emergency and elective surgery. J Reprod Med 1999;44:279.

[16]Leserowitz GS. Managing ovarian masses during pregnancy. Obstet Gynecol Surv 2006; $61: 463$
[17]Bernhard LM, Klebba PH, Gray DL, Mutch DG. Predictors of persistence of adnexal masses in pregnancy. Obstet Gynecol 1999; 93:585.

[18]Whitecar MP, Turner S, Higby MK. Adnexal masses in pregnancy: a review of 130 cases undergoing surgical management. Am J Obstet Gynecol 1999;181:19

[19]Yakasai IA, Bappa LA. Diagnosis and management of adnexal masses in preganancy. $\mathbf{J}$ Surg Tech Case Rep 2012;4:79.

[20]Sherard GB 3rd, Hodson CA, Williams HJ, et al. Adnexal masses and pregnancy: a 12year experience. Am J Obstet Gnecol 2003;189:358.

[21]Zanetta G, Mariani E, Lissoni A, et al. A prospective study of the role of ultrasound in the management of adnexal masses in pregnancy. BJOG 2003; 110:578. 